\title{
Transformation of land resources as a result of anthropogenic impact
}

\author{
Elena Sivak $^{1, *}$, and Svetlana Volkova $^{1}$ \\ ${ }^{1}$ Kursk State Agricultural Academy, 70, Karl Marx Street, 305021, Kursk, Russia
}

\begin{abstract}
The article examined temporary matrices of transformation of soil processes related to the activity of microorganisms and humus in comparison with maternal matrices depending on age characteristics of man. The resulting match in value and productivity, from one year to 15 years, from 15 years to 40 years, from 40 years to 65 years, led to the idea of comparing human growth with soil zonal; Weights with indicators of activity of microbiological soil processes; Intelligence with active humus; Age with dynamics of development of temporary matrices on humus and microbiological activity. The dynamics of transformation of organic substances of upper soil horizons under the influence of agricultural use according to the general humus, taking into account their genesis, covers the period from 15 to 46 years; And on microbiological processes from 3 to 66 years. The conclusion is that the identification of human development with the development of soil-formation processes depending on their genesis brings us closer to a true understanding of our appearance, as a species possessing the power that can change the surrounding world through the noosphere in accordance with certain standards of transmutation.
\end{abstract}

\section{Introduction}

Analyzing the intensity and direction of microbiological processes for the arable and its corresponding layer of the main stock soils [1] we see confirmation of the exponential law of micro-organism reproduction, which should be taken into account in determining the time of ecosystem development [2-10].

A review of foreign literature shows interest in natural-anthropogenic soil evolution [11-20] related to the effects of potassium sulfate and certain iron fertilizers on the physical and chemical properties of soils [12]; Forms of soil nitrogen due to soil acidity [14]; Studying the activity of soil enzymes under the influence of crop fertilization [20] and other transformations [13] related to the culturing of soil formation processes affecting the acceleration of the capture of living matter from the environment, which in turn lead to enrichment or depletion of vegetation cover, related to the ecological transformation of ongoing technological transformations and zone features of soils [15-22].

*Corresponding author: elena.sivak.77@mail.ru 


\section{Materials and methods}

Soils were considered as a material: dart-subtitle (DP), light grey forest (SS), black earth typical (CHT), dark chestnut (TC), red-yellow ferrallite (KF). With appropriate gradation: celine (C), forest (L), patchwork (D), cultured patchwork (OP). Briefly defined by a matrix with indicators of activity of microbiological processes, which vary vertically from 0.64 (pastille) to 36.38 (cultured pastille) million microorganisms per 1 ha of absolutely dry soil [2].

\begin{tabular}{|c|c|c|c|}
\hline & $\mathrm{C}$ & $\mathrm{P}$ & OP \\
\hline DP & 1,32 & 0,64 & 2,9 \\
\hline SS & 2,31 & 2,28 & 3,83 \\
\hline CHT & 9,40 & 8,73 & 9,61 \\
\hline $\mathrm{TC}$ & 10,27 & 10,58 & 14,82 \\
\hline $\mathrm{KF}$ & 20,26 & 31,60 & 36,38 \\
\hline
\end{tabular}

For the sake of clarity, the graph of the dynamics of the described processes depending on anthropogenic impact by soil zonal is of the form (Figure 1) [9].

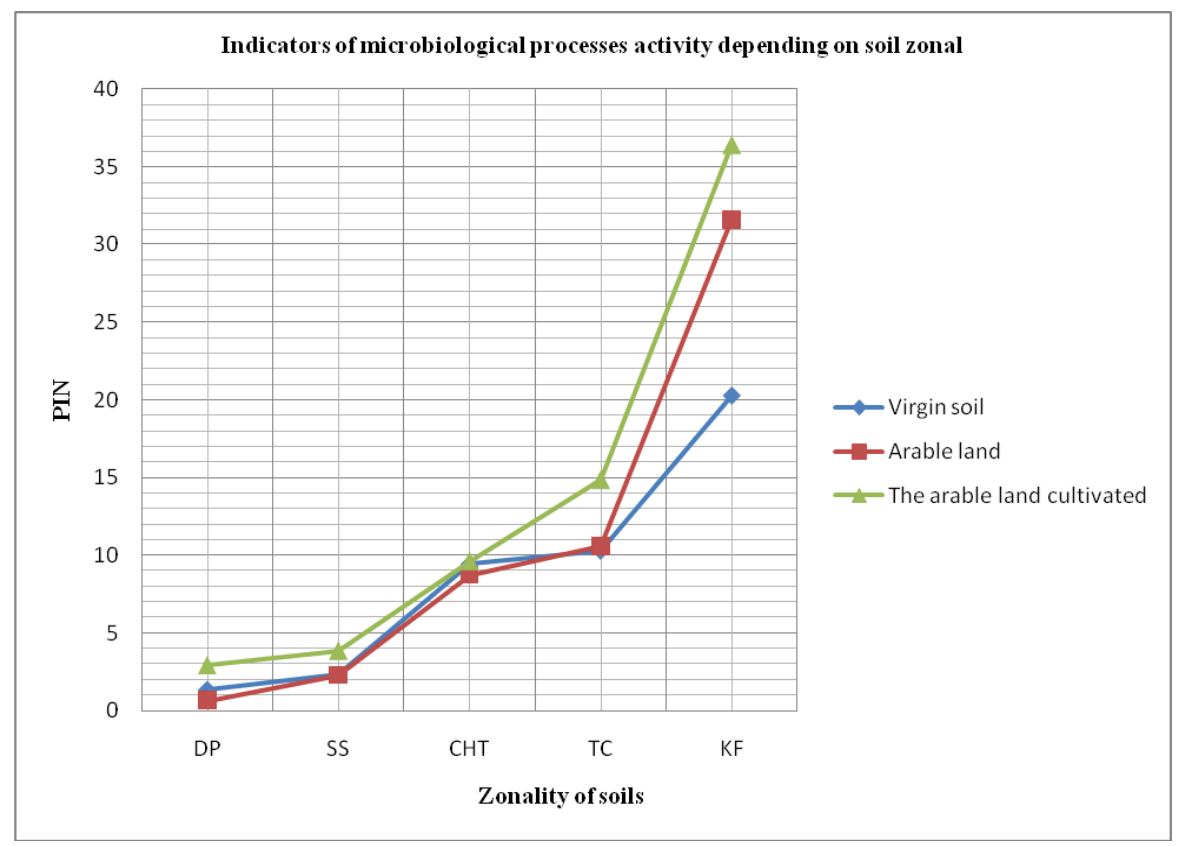

Fig. 1. Indicator of activity of microbiological processes (PIN) depending on soil zonal.

The method is Phelps 'law, namely:

$$
Q(t)=Q(0) e^{p t}
$$

where $\mathrm{Q}(\mathrm{t})$ is the number of microorganisms after time $\mathrm{t}$;

$\mathrm{Q}(0)$ is the number of microorganisms at the initial moment $t=0$;

$\mathrm{P}$ is the percentage of increase per unit of time in question;

$\mathrm{t}$ is the time period for considering the development of the reproduction process;

$\mathrm{e}$ - exhibitor $(\mathrm{e} \approx 2.72)$ 


\section{Results}

We will find the value $\mathrm{p} \cdot \mathrm{t}$ (growth of microorganisms), knowing their quantity at the initial moment of time $\mathrm{Q}(0)$, before the rake culturing and after a certain period $t$, knowing their quantity and $\mathrm{Q}(\mathrm{t})$. And we will build a matrix of development time from one state of the soil to another.

From formula (2) we obtain:

$$
\begin{gathered}
e^{p t}=\frac{Q(t)}{Q(0)} ; \quad p=\frac{\Delta Q}{100 \%} ; \quad \Delta Q=Q(t)-Q(0) . \\
p t=\ln \frac{Q(t)}{Q(0)} ; \\
t=\frac{1}{p} \ln \frac{Q(t)}{Q(0)} .
\end{gathered}
$$

The results of calculation according to formula (3) will be executed in the form of matrix (4) for further discussion: growth (p) for the whole time of Celin transformation cultured pasha (the first column in matrix (4)) and pasha - cultured pasha (the second column in matrix (4)).

$$
\begin{aligned}
& C-O P \quad P-O P \\
& \left.\begin{array}{c|cc}
D P & 0,016 & 0,023 \\
S S & 0,010 & 0,011 \\
C H T & 0,002 & 0,009 \\
T C & 0,046 & 0,042 \\
K F & 0,152 & 0,045
\end{array}\right) \text {; }
\end{aligned}
$$

Comparative analysis shows along the horizontal lines, i.e. the growth of microorganisms, namely the rate of their reproduction differs in times for blackness of typical (4.5 times) and only in the fourth and fifth lines the difference begins, but back, i.e. the growth of microorganisms goes faster on C-OP than P-OP. The reverse pattern shows a steeper vertical difference in both the initial data (matrix 1) and the increment in the first column. Zonal is the prevailing factor.

At that, the index of increment by the share of microorganisms is the least for blackness of typical (the third line of matrix (4)).

The time matrices of transition from one soil state to another under the action of anthropogenic factor from the state of celine to ocultural soils taking into account their zonality are reflected in the matrix (5), namely from $\sim 4$ years $(\mathrm{CP})$ to $\sim 50$ years $(\mathrm{CP})$ or from $\sim 3$ years $(\mathrm{CP})$ to 66 years $(\mathrm{CP})$, respectively.

$$
\left.\begin{array}{c|cc}
C-O P & P-O P \\
D P & 49,382 & 65,560 \\
S S & 36,343 & 34,424 \\
C H T & 11,046 & 10,657 \\
T C & 7,976 & 8,027 \\
K F & 3,596 & 2,959
\end{array}\right)
$$


By total humus (\%) matrix (6) we see that its greatest value in black earth is typical.

Total humus transformation matrix, \% [1] by soil zonal.

$$
\begin{aligned}
& \begin{array}{lll}
C & P & O P
\end{array} \\
& \left.\begin{array}{c|ccc}
D P & 2,21 & 2,03 & 2,65 \\
S S & 3,1 & 1,68 & 2,79 \\
C H T & 7,05 & 5,25 & 5,65 \\
T C & 4,63 & 3,11 & 3,30 \\
K F & 6,01 & 2,97 & 4,56
\end{array}\right)
\end{aligned}
$$

\section{Discussion}

Analyzing the remaining indicators of matrices (4) - (6) we conclude that if we rank soils by humus, we will get matrix (7). The resulting matrix, ranked by humus, in which the columns correspond to the lands: 1 - celine, 2 - patchwork, 3 - cultured patchwork.

\begin{tabular}{c|ccc|c}
1,3 & 1 & 2 & 3 & 2 \\
$D P$ & 2,21 & 1,68 & 2,65 & $S S$ \\
$S S$ & 3,1 & 2,03 & 2,79 & $D P$ \\
$T C$ & 4,63 & 2,97 & 3,30 & $K F$ \\
$K F$ & 6,01 & 3,11 & 4,56 & $T C$ \\
$C H T$ & 7,05 & 5,25 & 5,65 & $C H T$
\end{tabular}

In which, in the first column the third, fourth and fifth lines are changed places, respectively, in the second column the 2 nd line with the first, the third with the fifth and in the third column there is a pattern similar to the first. By examining the matrix (7), we see that the pattern of increase by humus in blackness is typical stable in all three variants, i.e. its greatest value regardless of the land. We will compile the dynamics of transformation of organic matter of upper soil horizons under the influence of agricultural use according to the general humus. Similarly to the fifth matrix, taking the changes of the humus of the general in formula (3) and obtaining the matrix (8).

$$
\left.\begin{array}{c|cc}
D P & 41,265 & 42,988 \\
S S & 33,987 & 45,689 \\
C H T & 15,812 & 18,357 \\
T C & 25,461 & 31,210 \\
K F & 19,042 & 26,966
\end{array}\right)
$$

Comparing (5) and (8) the matrices show a tendency to decrease in the time of transition of the intensity of microorganisms of processes related to the number of microorganisms taking into account the main zonal soils. By humus, the situation is much more complicated and wave-like (Figure 2). 


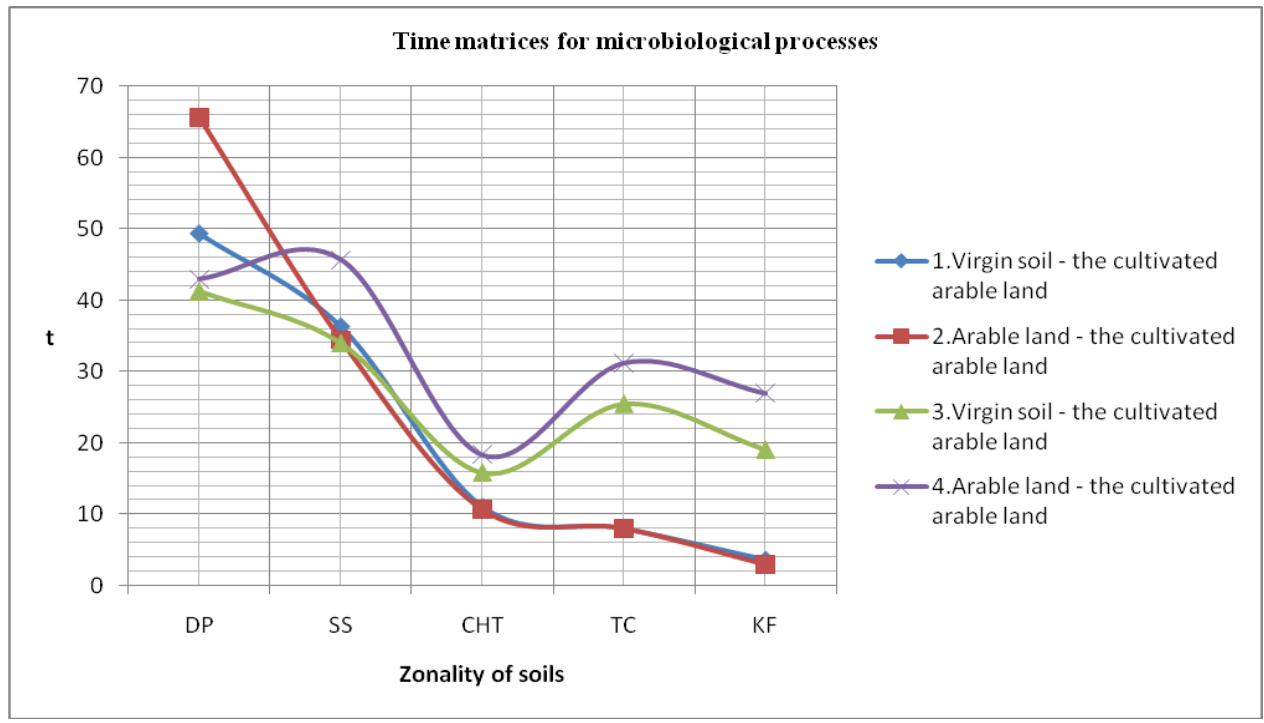

Fig. 2. Time matrices by microbiological processes: 1, 2; By humus in general: 3,4 .

The humus is active, $\%$

$\left.\begin{array}{c|ccc}D P & 0,3 & 0,65 & 1,01 \\ S S & 0,64 & 0,61 & 1,09 \\ C H T & 1,75 & 2,1 & 2,35 \\ T C & 1,6 & 1,5 & 1,76 \\ K F & 0,12 & 0,297 & 0,299\end{array}\right)$

Matrix (9) is obtained, by humus active.

Using the matrix data (9) as the basis and applying the formula (3), the elements of the matrix (10) characterizing the transition time from celine to pasha ( 1 column), from pasha to pasha cultured ( 2 column), from celine to pasha cultured ( 3 column) are obtained. The lines characterize the zonal of soils. The least time of interreversion is in black of typical and dark chestnut soils, 45 to 52 years and 60 to 65 years, respectively.

\begin{tabular}{|c|c|c|c|}
\hline & $C-P$ & $P-O P$ & $C-O P$ \\
\hline$D P$ & 220,91 & 122,43 & 170,98 \\
\hline$S S$ & 160,03 & 120,93 & 118,33 \\
\hline CHT & 52,09 & 44,99 & 49,13 \\
\hline$T C$ & 64,54 & 61,48 & 59,57 \\
\hline$K F$ & 509,05 & 335,57 & 510,03 \\
\hline
\end{tabular}

The graphs of dynamics of the described processes depending on anthropogenic impact have the form (Figure 3) [10].

Due to acceleration of metabolic processes, such behavior of active humus under the influence of heat, moisture, biotic factors, small biological circulation, has more revolutions. At first the amount of active humus increases, and then under the influence of heat, moisture, other biological factors inherent in zones with dark chestnut and red-yellow ferrolite soils begin to decrease. But in general, comparing the amounts from celine, to pasha cultured increases from $4.41 \%$ to $5.16 \%$ (pasha) and $6.54 \%$ (pasha cultured). 


\section{Dependence of $\mathrm{Ag}=\mathbf{f}$ (PIN)}

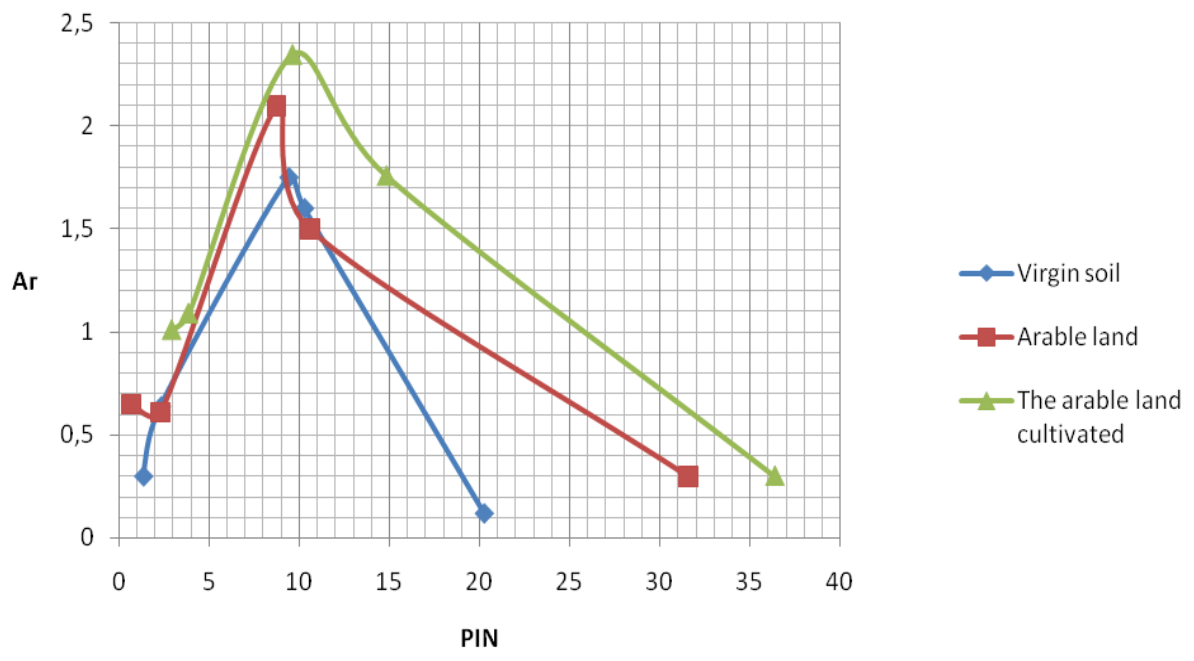

Fig. 3. The dependence $A g=f(P I N)$.

Soil age is similar to human age. There is a distinction between the absolute age from the beginning of soil formation to the present moment and the relative age - the degree of development of this soil, about the person in this sense is said "smart not by years." At the same absolute age, soils differ dramatically in their development due to the different soil formation rate and degree of its manifestation in this soil, as confirmed by our studies highlighting: Young (16 to 46 years old) matrix (8) and mature (49 to 70 years old) matrix (5) and very mature (70 to 600 years old) matrix (10) with a clear expression of all genetic horizons defining a particular soil type. In our work, we have considered time matrices as functions of soil formation processes. They are, as our research shows [21.22], nonlinear in nature, which is confirmed by this work.

$\mathrm{T}=\mathrm{f}(\mathrm{P})$, where $\mathrm{T}$ is time, $\mathrm{P}$ is soil, having taken the remaining values equal to certain values according to the genesis of soils (climate, organisms, mother rocks, relief).

Conclusions. Our studies show that by the activity of organisms in the soil-forming process, the lower border is occupied by the patchwork, the upper cultured patchwork, and between them celine and only from the dark chestnut soil to the red-ferrallite boundary of activity the lower border becomes celin, and the middle patchwork changes (Figure 1).

Matrices of temporal development of soils by humus in general coincide with matrices of human development from birth to maturity with pronounced peaks of age characteristics (Figure 2).

As humus activity (matrix 9) increases, the transition time from one soil state to another (matrix 10) decreases, i.e., there is an inverse dependence of humus activity on transformation time, which confirms the correlation coefficient rtAg $=-0.8$.

Therefore, we believe that the picture will be more complete if we consider the economic activity of man on earth, that is, to consider temporary matrices in terms not only of soil formation, but also of man-made factor, which is the result of insurmountable circumstances involved in soil formation processes and actively affecting their development in accordance with accepted standards of typical soils. 


\section{References}

1. S.N. Volkova, D.V. Muha, Modeling and prediction of evolutionary processes in socio-ecological systems (KGSHA, Kursk, 2019)

2. V.D. Muha, Natural-man-made soil evolution (general patterns and zonal features) (Ear, Moscow, 2014)

3. S.N. Volkova, D.V. Muha, Reports of the Russian Academy of Agricultural Sciences 1, 17 (2016)

4. S.N. Volkova, D.V. Muha, Reports of the Russian Academy of Agricultural Sciences 1, 29 (2017)

5. V.D. Muha, S.N. Volkova, D.V. Muha, Materials of the International Scientific and Practical Conference, 49-51 (Kursk, 2013)

6. S.N. Volkova, VD. Muha, E.E.Volkova, D.N. Muha, Modeling of universal interaction of evolutionary processes in social and ecological systems: monograph (KGSHA, Kursk, 2009)

7. S.N. Volkova, O.I. Majorov, E.E. Siwak, M.A. Myasoedova, S.N. Potemkin, Journal of KGSHA 2, 78-80 (2012)

8. V.A. Sysuev, A.V. Aleshkin, Yu.V. Sichugov, A.Yu. Yusupov, Theoretical and applied ecology 4, 24-29 (2018)

9. E.A. Mamontova, E.N. Tarasova, A.A. Mamontov, E.V. Ivanov, Theoretical and applied ecology 1, 43-52 (2018)

10. I.G. Shirokikh, Ya.I. Nazarova, A.A. Shirokikh, T.Ya. Ashikhmina, Theoretical and applied ecology 1, 80-87 (2018)

11. E.V. Sokolskaya, B.I., Kochurov, Yu.A. Dolgov, V.A. Lobkovsky, Theoretical and applied ecology 2, 26-34 (2018)

12. T.A. Trifonova, N.V. Mishchenko, Zh.D. Petrosyan, Soil Science 2, 152-164 (2020)

13. A.A. Dymov, E.Yu. Milanovsky, Soil Science 2, 178-187 (2020)

14. N.I. Sukhanova, S.Ya. Trofimov, A.L. Stepanov, A.V. Kiryushin, Soil Science 2, 199209 (2020)

15. A.K. Khodzhaeva, A.V. Sutilovic, S.V. Gubin, A.V. Lupachev, Soil Science 2, 210218 (2020)

16. A.V. Bogorodskaya, A.S. Shishikin, Soil Science 1, 119-130 (2020)

17. L.A. Ovsepyan, I.N. Kurganova, V.O. Lopez de Guerenu, A.V. Rusakov, Ya.V. Kuzyakov, Soil science 1, 56-68 (2020)

18. T.T. Efremova, S.P. Efremov, N.In. Melent'eva, A.F. Lavrov, Soil science 8, 923-934 (2019)

19. M.B. Yuldasheva, O.I. Yuldashev, Computational Mathematics and Modeling 30.3, 267-284 (2019)

20. A.G. Belov, Computational Mathematics and Modeling 30.3, 285-294 (2019)

21. A.V. Voronenko, A.A. Shchurova, Computational Mathematics and Modeling 30.1 (2019)

22. V.I. Dmitriev, I.S. Barashkov, Computational Mathematics and Modeling 30.1, 55-67 (2019) 